\title{
TESTICULAR HISTOPATHOLOGICAL DIAGNOSIS AS A PREDICTIVE FACTOR FOR RETRIEVING SPERMATOZOA FOR ICSI IN NON-OBSTRUCTIVE AZOOSPERMIC PATIENTS
}

\author{
SIDNEY GLINA, JONATHAS B. SOARES, NELSON ANTUNES JR, ANDREA G. GALUPPO, \\ LUCERO B. PAZ, ROBERTA WONCHOCKIER
}

Human Reproduction Unit, Albert Einstein Jewish Hospital, Sao Paulo, SP, Brazil

\begin{abstract}
Objective: Histological testicular pattern has a predictive role in the possibility of finding spermatozoa for ICSI in cases of non-obstructive azoospermia because some individuals could show residual spermatogenic sites in the testis. The aim of this study was to evaluate the sperm retrieval rate in each of the histopathological groups (hypospermatogenesis-Hypo, spermatogenic maturation arrestMA, Sertoli cell only-SCO and testicular hyalinization) in patients assisted in our clinic.

Materials and Methods: Retrospective study from March 1997 to October 2002. We analyzed 14 patients with mean age of $34.3 \pm 0.7$, with non-obstructive azoospermia. All patients were submitted to previous diagnostic biopsy $(\mathrm{Bx})$ elsewhere and came to our institution for treatment. After an average of 12 months $(8-20)$, they were submitted to a new Bx procedure to retrieve sperm.

Results: Previous diagnostic Bx showed the following histopathological results: 5 patients with MA, 4 with Hypo and 5 SCO. In the following Bx (for sperm retrieval) spermatozoa was found in $33 \%$ of the procedures in patients with MA, 50\% in patients with Hypo and $40 \%$ of the procedures in patients with SCO.

Conclusion: Previous diagnostic Bx can help in patient counseling concerning the result of sperm retrieval.
\end{abstract}

Key words: azoospermia; testis; biopsy; histopathology; sperm injections, intracytoplasmic

Int Braz J Urol. 2005; 31: 338-41

\section{INTRODUCTION}

The advent of intracytoplasmic sperm injection (ICSI) has represented a major breakthrough in the treatment of infertile men. Men with nonobstructive azoospermia could be biological fathers due to the possibility of testicular sperm extraction $(1,2)$. High rates of sperm retrieval in ICSI procedures performed with sperm retrieved by testicular biopsy have been described (3).

Finding sperm in the testis in cases of nonobstructive azoospermia vary according to the histopathological pattern of the testis (4). The most frequent histopathological patterns are: hypospermatogenesis (Hypo), spermatogenic maturation arrest (MA), Sertoli cell only (SCO) and testicular hyalinization (5).

The possibility of finding sperm in SCO cases is around $20 \%$, in Hypo patients it is $80 \%$ and in MA it is around $50 \%(5)$. The aim of this work was to evaluate the retrieval sperm frequency in each of the histopathological groups in our institution.

\section{MATERIALS AND METHODS}

This was a retrospective study performed between March 1997 and October 2002. We analyzed 
14 patients with mean age of $34.3 \pm 0.7$ years with nonobstructive azoospermia who had undergone diagnostic testicular biopsies in other centers. 12 months after the first biopsy a new procedure was performed to retrieve spermatozoa and in 12 cases the ICSI was performed simultaneously. The groups were classified according to the biopsy diagnosis: spermatogenic maturation arrest (MA), hypospermatogenesis (Hypo) and Sertoli Cell Only (SCO). Two patients underwent ICSI - one from the hypo group and one from the MA group. We performed 2 ICSI cycles and 2 biopsies each, resulting in 16 procedures for sperm retrieval.

\section{Sperm Collection by Testicular Biopsy (TESE)}

The testicular biopsy was performed after the spermatic cord was blocked using local anesthesia, specifically $2 \%$ Xylocaine without epinephrine or under endovenous sedation. Using a number 11 cold scalpel, a longitudinal incision was performed in the scrotum order to expose the testicles. Testicular compression was conducted, leading to glandular tissue herniation that was excised. The tunica albuginea was closed with monofilament nylon 5-0 sutures. Three fragments of glandular tissue were retrieved when the testicular volume was smaller than $15 \mathrm{cc}$, and 6 fragments were removed in the other cases (Figure-1). When no sperm was found in the first biopsied testicle, the procedure was performed on the other. All fragments were weighed in an OHAUS analytical standard electronic balance (USA).
The material collected was carefully dissected in a Petri dish containing human tubal fluid modified medium (HTFmod-Irvine Scientific, USA) with 2 cold scalpels (number 22). Then the material was analyzed to identify the presence of spermatozoa under optical microscope (x400). The material was kept in a sterile Eppendorf at $37^{\circ} \mathrm{C}$ for 3-4 hours to allow the sperm to migrate to the medium surface.

\section{RESULTS}

The average time between the previous diagnostic biopsy and the sperm retrieval for ICSI was 12 months (8-20 months). The average weight of testicular tissue excised for sperm retrieval was $0.0659 \mathrm{~g}$ (0.0044 to 0.1761$)$. Sperm finding at retrieval biopsies according to the histopathological diagnosis are shown in Table-1. One patient who underwent two biopsies (sperm retrieval) for 2 different ICSI cycles had a previous biopsy with histopathological diagnosis of hypospermatogenesis and in both biopsies sperm were found. The other patient (MA group) showed one biopsy with the presence of sperm and another one with no sperm found. The average break time between those biopsy procedures was 12 months ( 8 to 20). After all ICSI procedures, there were 3 pregnancies ( 1 in the MA group and 2 in the Hypo). The sperm retrieval biopsy results are shown in Table- 1 according to the histopathological diagnosis.

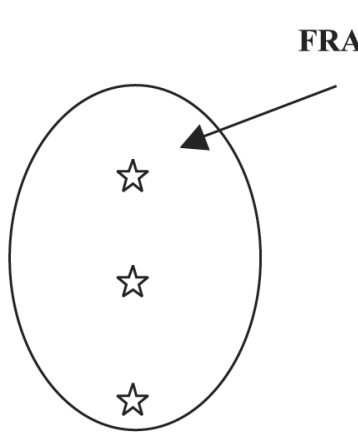

A

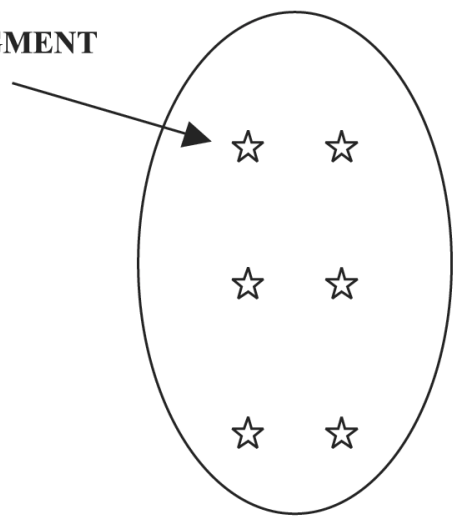

B

Figure 1 - Diagram of fragments retrieval according to testicle size. A) Testis volume < $15 \mathrm{cc}$; B) Testis volume >15 cc. 
Table 1 - Sperm retrieval biopsy results according to histopathological diagnosis.

\begin{tabular}{lcccc}
\hline & MA & Hypo & SCO & Total \\
\hline Total patients & 5 & 4 & 5 & 14 \\
Total procedures & 6 & 5 & 5 & 16 \\
Presence of Spz & 2 & 3 & 2 & 7 \\
\hline
\end{tabular}

Spz $=$ spermatozoa, $M A=$ spermatogenic maturation arrest, Hypo = hypospermatogenesis, $S C O=$ Sertoli cell only.

\section{COMMENTS}

Residual spermatogenesis sites in the testis can be found in patients with non-obstructive azoospermia; however there are as yet no defined prognostic parameters for this finding (6). Data such as testicular volume, FSH serum concentration and presence of associated male pathologies cannot be used as predictive factors of success (4). Previous data showed that age and serum FSH levels failed in foresee the presence or absence of spermatozoa in the testicular biopsy $(7,8)$. However, techniques such as molecular markers and RT-PCR are useful in predicting the presence of testicular sperm (9-11).

Brugo-Olmedo et al. (12) showed that the Binhibin plasma levels, as a Sertoli Cell activity measurement, could be related to the chance of retrieval spermatozoa in patients with non-obstructive azoospermia. Patients with biopsies where sperm was found presented significantly high levels. However other studies failed to correlate B-inhibin levels and the chances of sperm retrieval in the testis $(13,14)$. According to Schoor et al. (2), a diagnostic testicular biopsy is one parameter for determining the testicular histopathology pattern and apparently it is the strongest indicator to foresee the possibility of finding sperm in the testis in the sperm retrieval procedure $(7,8)$. Several studies have suggested that the presence of one focus of elongated spermatides or spermatozoa in a diagnostic biopsy is related to high sperm retrieval rates for ICSI $(4,15)$.

Controversial results have been shown in nonobstructive azoospermia patients as to what is the best technique for sperm retrieval. According to Schoor et al. (2), a testis biopsy could promote scars that make the following biopsy procedures more difficult. Sousa et al. (4) consider testicular biopsy the best method for sperm retrieval in non-obstructive azoospermic patients, considering that in testicular atrophy cases percutaneous sperm aspiration was not able to provide enough material for ICSI. Considering that spermatogenesis recovery after a biopsy procedure is slow, it is essential to avoid unnecessary biopsies (16). A maximum of 3 procedures must be performed and, whenever possible, they should be associated with cryopreservation techniques $(4,2)$. This care reduces the risk of complications without impairing fertilization and pregnancy rates (16). In our patients showing normal testicular volume, 6 tissue fragments were retrieved from different sites and 3 fragments showed reduced testicle volume. Schlegel (17) states that the micro-dissection technique was the one that presented the best retrieval results when compared with multiple biopsy techniques, however this is not a consensus yet.

There is a discrepancy between our results and the literature on the likelihood of obtaining sperm in non-obstructive azoospermic patients; the percentage of our patients with positive sperm retrieval according to histological testicular pattern was 50\% in patients with hypospermatogenesis, 33\% in patients with spermatogenic arrest and $40 \%$ in patients with SCO against $80 \%, 50 \%$ and $20 \%$, respectively in the literature (5). Probably the main reason for that was the small size of our sample.

Although histopathological testicular pattern plays a role in the probability of finding sperm in subsequent sperm retrieval procedures, we do not recommend it without simultaneous cryopreservation. Although this sample size was limited, it is still 
important that other authors publish their data in order to allow a definition about what is the real chance of sperm retrieval in each histopathological pattern.

\section{REFERENCES}

1. Van Steirteghem AC, Nagy Z, Joris H, Liu J, Staessen C, Smitz J, Wisanto A, Devroey P: High fertilization and implantation rates after intracytoplasmic sperm injection. Hum Reprod. 1993; 8: 1061-6.

2. Schoor RA, Elhanbly S, Niederberger CS, Ross LS: The role of testicular biopsy in the modern management of male infertility. J Urol. 2002; 167: 197-200.

3. Silber SJ, Van Steirteghem AC, Devroey P: Sertoli cell only revisited. Hum Reprod. 1995; 10: 1031-2.

4. Sousa M, Cremades N, Silva J, Oliveira C, Ferraz L, Teixeira da Silva J, et al.: Predictive value of testicular histology in secretory azoospermic subgroups and clinical outcome after microinjection of fresh and frozen-thawed sperm and spermatids. Hum Reprod. 2002; 17: 1800-10.

5. Cedenho AP, Bortoluzzo C, Vieira M: What is Important in Evaluation of Infertile Men. In: First Brazilian Consensus in Male Infertility. Brazilian Society of Urology. Sao Paulo, BG Cultural. 1999; chapt. 3, p. 17. [in Portuguese]

6. Silber SJ, Van Steirteghem AC, Liu J, Nagy Z, Tournaye H, Devroey P: High fertilization and pregnancy rate after intracytoplasmic sperm injection with spermatozoa obtained from testicle biopsy. Hum Reprod. 1995; 10: 148-52.

7. Mercan R, Urman B, Alatas C, Aksoy S, Nuhoglu A, Isiklar A, Balaban B: Outcome of testicular sperm retrieval procedures in non-obstructive azoospermia: percutaneous aspiration versus open biopsy. Hum Reprod. 2000; 15: 1548-51.

8. Vicari E, Grazioso C, Burrello N, Cannizzaro M, D'Agata R, Calogero AE: Epididymal and testicular sperm retrieval in azoospermic patients and the outcome of intracytoplasmic sperm injection in relation to the etiology of azoospermia. Fertil Steril. 2001; 75: 215-6.

9. Friel A, Houghton JA, Glennon M, Lavery R, Smith T, Nolan A, et al.: A preliminary report on the implication of RT-PCR detection of DAZ, RBMY1, USP9Y and Protamine- 2 mRNA in testicular biopsy samples from azoospermic men. Int J Androl. 2002; 25: 59-64.

10. Patrizio P, Ricci SM, Tomaszewski JE, Hecht NB: Identification of meiotic and postmeiotic gene expression in testicular tissue of patients histologically classified as Sertoli cell only. Fertil Steril. 2000; 74: 785-90.

11. Song GJ, Lee H, Park Y, Lee HJ, Lee YS, Seo JT, et al.: Expression pattern of germ cell-specific genes in the testis of patients with non-obstructive azoospermia: usefulness as a molecular marker to predict the presence of testicular sperm. Fertil Steril. 2000; 73: 1104-8.

12. Brugo-Olmedo S, De Vincentiis S, Calamera JC, Urrutia F, Nodar F, Acosta AA: Serum inhibin B may be a reliable marker of the presence of testicular spermatozoa in patients with non-obstructive azoospermia. Fertil Steril. 2001; 76: 1124-9.

13. von Eckardstein S, Simoni M, Bergmann M, Weinbauer GF, Gassner P, Schepers AG, et al.: Serum inhibin B in combination with serum follicle-stimulating hormone (FSH) is a more sensitive marker than serum FSH alone for impaired spermatogenesis in men, but cannot predict the presence of sperm in testicular tissue samples. J Clin Endocrinol Metab. 1999; 84: 2496-501.

14. Vernaeve V, Tournaye H, Schiettecatte J, Verheyen G, Van Steirteghem A, Devroey P: Serum inhibin B cannot predict testicular sperm retrieval in patients with non-obstructive azoospermia. Hum Reprod. 2002; 17: 971-6.

15. Rosenlund B, Kvist U, Ploen L, Rozell BL, Sjoblom P, Hillensjo T: A comparison between open and percutaneous needle biopsies in men with azoospermia. Human. Reprod. 1998; 13: 1266-71.

16. Dirnfeld L, Paz M, Yshai D, Calderon I, Lahav-Baratz S, Koifman M, et al.: The impact of early testicular sperm extraction or cryopreservation on the outcome of intracytoplasmic sperm injection - a randomized controlled study. J Assist Reprod Genet. 2003; 20: 205-9.

17. Schlegel PN: Testicular sperm extraction: microdissection improves sperm yield with minimal tissue excision. Hum. Reprod. 1999; 14: 131-5.

$$
\begin{array}{r}
\overline{\text { Received: July 17, } 2004} \\
\text { Accepted after revision: May 10, } 2005
\end{array}
$$

